\title{
Research of Supercapacitor Voltage Equalization Strategy on Rubber-Tyred Gantry Crane Energy Saving System
}

\author{
Chunhe CHANG ${ }^{1}$, Jiangping YANG ${ }^{1}$, Yu LI ${ }^{2}$, Zhongni ZHU ${ }^{1}$ \\ ${ }^{1}$ Air Force Radar Academy, AFRA, Wuhan, China \\ ${ }^{2}$ South-central University for Nationalities, SCUN, Wuhan, China \\ Email:cch1725@163.com
}

\begin{abstract}
A model for supercapacitor voltage equalization strategy is analyzed, and on this basis a supercapacitor voltage equalization method for Rubber Tyred Gantry Crane (RTG) energy saving system is proposed, namely active voltage equalization method based on Buck-Boost converter. The equalizing speed of the proposed method is fast. Firstly, the working principle and process of the voltage equalization circuit is analyzed in detail. In addition, design of active voltage equalization circuit parameters and control strategy are given. Finally, simulation analysis of the series connection of supercapacitors module is performed. Results show that this method for equalizing voltage can avoid over-voltage of each cell and possess practicable and high value for supercapacitor RTG energy saving system.
\end{abstract}

Keywords: supercapacitor, energy saving, rubber tyred gantry crane (RTG), voltage sharing, sesign of active equalization circuit

\section{Introduction}

Supercapacitor is a novel energy storage device based on the principle of the double layer-electrolyte capacity, which has many merits such as long lifetime, high efficiency, fast dynamic response, etc. So it is a power storage technology that has a bright future in power storage development. The power driver system in energy -saving RTG is composed of the diesel generator set, power balance system (made by supercapacitors), controller and the rising electromotor. Supercapacitors are used for storing energy from which electromotor generates and brakes energy when the load is fallen down, the supercapacitors release the energy which has been stored when the load is raising. Thus the original energy which is consumed by the braking resistance is recycled totally, then the purpose of energy saving and environment protection is realized.

Due to the lower voltage of a single supercapacitor, generally speaking, the series and parallel connection of supercapacitors form the energy storage module to meet the energy storage capacity and higher voltage requirements. However, the operational voltage of supercapacitors is different, and a local over-voltage can appear over one or several supercapacitors, which would affect the lifetime and reliability of the system. Therefore, it is essential and critical to research and realize supercar- pacitors voltage equalization technology for improving the supercapacitors power storage technology.

The present supercapacitors voltage equalization technology mainly includes zener diode type, switch resistor type, switch capacitor type, inductor type, forward converter type and flyback converter type voltage equalization circuits, etc. The switching resistor type and voltage-regulator diode type voltage equalization circuits consume amount of energy because of utilizing energy-consuming devices, so the system has lower efficiency and poorer reliability [1]. The switching capacitor type and inductor type voltage equalization circuits have ineffective energy flowing, especially when the two adjacent supercapacitors voltage difference is very closer or when much more supercapacitors are in series connection, balancing speed will be slower [2] Forward converter type and flyback converter type voltage equalization circuit have a higher efficiency, but they are not attractive, because they also have many demerits such as complicated magnetic circuit, big volume, difficult extended winding and large voltage equalization error, etc [3]. In view of the existing problems of the above voltage equalization methods, the paper proposes an active voltage equalization method based on the principle of Buck-Boost converter, this method can transfer energy from the high-voltage supercapacitors to the low-voltage ones through the converter rapidly, and it has the character of the low 
energy loss and the high equalizing speed in the process of charging and discharging.

\section{Analysis of Voltage Equalization Model}

The supercapacitor charging equalizing model is shown in Figure 1. On the basis of the paper [4], the model is further analyzed in detail. Assuming the value of two optional supercapacitors are $C_{1}$ and $C_{2}, d_{1}$ and $d_{2}$ are capacity deviation values of the supercapacitors $C_{1}$ and $C_{2}$ respectively, we define the values of $C_{1}$ and $C_{2}$ as below:

$$
C_{1}=C\left(1+d_{1}\right), \quad C_{2}=C\left(1+d_{2}\right)
$$

where $C$ is the reference value for capacitors.

If two supercapacitors are connected in series, both of the initial voltage values are zero, a constant current $I$ charges supercapacitors, the voltage difference during the same time $t$ is defined as following:

$$
\Delta U=\left|U_{c 1}-U_{c 2}\right|=\frac{\Delta C}{C_{1} C_{2}} I t
$$

where $\Delta C$ is the capacity difference between $C_{1}$ and $C_{2}$.

If two supercapacitors are connected in series, both of the initial voltage values are zero, when different constant currents $I_{c 1}$ and $I_{c 2}$ charge supercapacitors, the voltage difference during the same time $t$ can be expressed as in (3):

$$
\Delta U=\left(\frac{I_{c 1}}{C_{1}}-\frac{I_{c 2}}{C_{2}}\right) t
$$

Substituting (1) into (3) when the voltage difference is zero, the relation between charging current and the supercapacitor capacity deviation can be obtained as follows:

$$
\frac{I_{c 1}}{I_{c 2}}=\frac{C_{2}}{C_{1}}=\frac{1+d_{1}}{1+d_{2}}
$$

If two supercapacitors are connected in series, both of the initial voltage values are zero, the supercapacitors voltage rise from zero to the upper voltage $U_{u}$ when

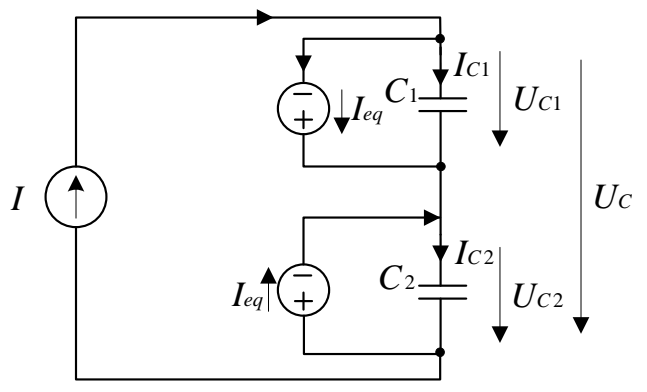

Figure 1. The supercapacitor charging equalizing model constant current charges supercapacitors, the two cells voltages can be, respectively, calculated as:

$$
U_{c 1}=\frac{1+d_{1}+d_{2}}{2+d_{1}+d_{2}} U_{c}, \quad U_{c 2}=\frac{1}{2+d_{1}+d_{2}} U_{c}
$$

where $U_{c 1}$ and $U_{c 2}$ are the voltages across the supercapacitors $C_{1}$ and $C_{2}$ at the end of charging and $U_{c}$ is the total voltage. Obviously, the two supercapacitors capabilities are same when $d_{1}=d_{2}=0$, thus, the capacitors voltages can be described as: $U_{c 1}=U_{c 2}=$ $U_{c} / 2=U_{u}$.

As Figure 1 is shown, assuming a current of equalizing current supply $I_{e q}=k_{j} I$ (both the two current supplies are reverse direction) is parallel connected with each capacitor side, $k_{j}$ is equalizing coefficient. Then, the charging current across $C_{1}$ and $C_{2}$ can be, respecttively, described as:

$$
I_{c 1}=I\left(1-K_{j}\right), I_{c 2}=I\left(1+K_{j}\right)
$$

Substituting (6) into (4), the ration between constant current source $I$ and the charging current $I e q$ can be expressed as follows:

$$
k_{j}=\frac{d_{2}-d_{1}}{2+d_{1}+d_{2}}
$$

Then, the two supercapacitors charging current can be, respectively, calculated as:

$$
I_{c 1}=\frac{2+2 d_{1}}{2+d_{1}+d_{2}} I, \quad I_{c 2}=\frac{2+2 d_{2}}{2+d_{1}+d_{2}} I
$$

Usually the supercapacitor capacity deviation $d$ is not zero, but it is a random value, which variable range is $-10 \% \sim+20 \%$. The relation between the equalizing current $I_{e q}$ and the charging current $I$ can be calculated from $d$ and (7) when the supercapacitors are in voltage equalization state.

$$
I_{e q} \geq 0.143 I
$$

From (3), it can be concluded that if the supercapacitors initial voltage is not zero, the voltage difference $\Delta U$ will decrease gradually, and $k_{j}$ value continues to increase, the voltage difference across the supercapacitors reduces more quickly.

\section{Active Voltage Equalization Circuit}

In view of the energy of the high-voltage supercapacitors is directly transferred to the low-voltage supercapacitors, this paper proposes an effective voltage equalization method-Active Circuit of Voltages Balance for the Series Supercapacitors. This method compares with the methods of "INDUCTION", and it is characterized by the low energy loss and the high equalizing speed in the process of charging and discharging. 


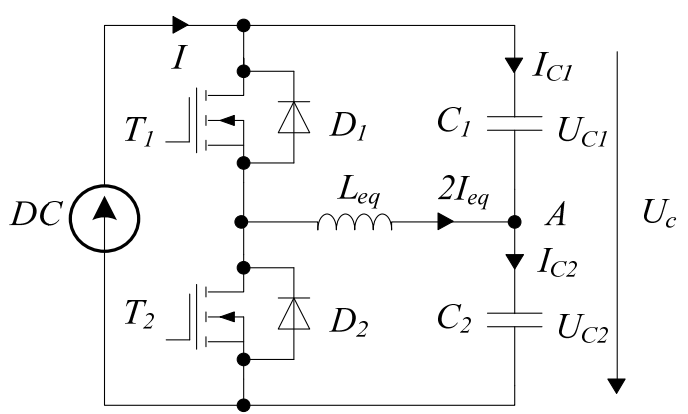

Figure 2. Active circuit of voltages equalization for two series supercapacitors
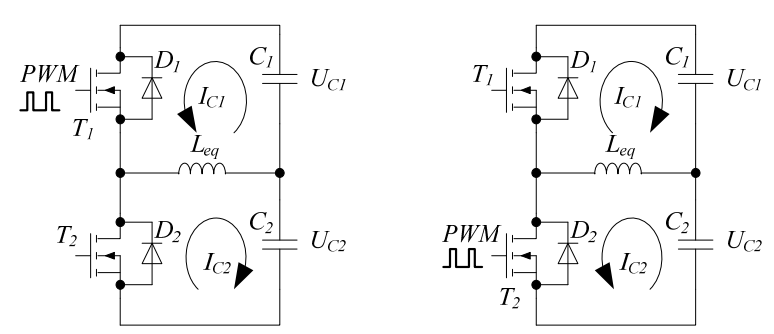

Figure 3. The principle circuit of active voltages balance

\subsection{The Basic Operational Principle}

As shown in Figure 6, switches $T_{1}, T_{2}$ are MOSFET; diodes $D_{1}, D_{2}$ are continued flow diodes; $L_{e q}$ is the energy storage inductor; $C_{1}, C_{2}$ are two adjacent series cells, respectively. A Buck-Boost converter can be connected with two adjacent cells of supercapacitors.

The basic operational principle is shown in Figure 7. As is shown in Figure 3(a) below, when $U_{c 1}>U_{c 2}$, a PWM drive signal is given to the switches, and switch $T_{2}$ is turned off and $T_{1}$ is turned on. While $T_{1}$ is on, supercapacitor $C_{1}$, switch $T_{1}$ and inductor $L_{e q}$ forms a loop circuit, whose current is $I_{c 1}$. The part of energy of supercapacitor $C_{1}$ transfers to inductor $L_{\text {eq }}$. While $T_{1}$ is off, supercapacitor $C_{2}$, inductor $L_{e q}$ and the diode $D_{2}$ forms a loop circuit, whose current is $I_{c 2}$. The energy of inductor $L_{e q}$ transfers to supercapacitor $C_{2}$. Similarly, as is shown in Figure 3(b) below, when $U_{c 1}<U_{c 2}$, switch $T_{1}$ is turned off and $T_{2}$ is turned on. The energy transfers from $C_{2}$ to $C_{1}$ until the voltages of the two supercapacitors are same.

\subsection{Analysis of Operation Process of Voltage Equalization}

According to the above principle of voltage equalization, and in order to analyze the operation process of the circuit, assume that the following items are satisfied:

1) It is assumed that the voltage of diode, the internal resistance of inductor, the on-resistance of the switch and the resistance of the circuit are all ignored, and supercapacitors are assumed to be ideal capacitors;

2) The circuit works in discontinuous conduction mode (DCM);

3) The capacity of the supercapacitor $C_{m}$ is less than that of $C_{m+1}$, that it is to say, the supercapacitor $C_{m}$ voltage is higher than $C_{m+1}$, where $m$ is positive integer;

4) Because of the large capacity of supercapacitors and high swtiching frequency, the supercapacitor can be seen as a voltage supply during a switching period.

Equivalent circuit of active voltage equalization system of two supercapacitors is shown in Figure 4. Referring to the above equivalent circuit, the operating process of the voltage equalization can be analyzed in detail as follows:

\subsubsection{Operation Mode $1\left(0 \leq t \leq D_{1} T\right)$}

At $t=0$, the switch $T_{m}$ is turned on, the diode $D_{m}$ is turned off. According to the above assumption, the Figure 4 can be equivalent to Figure 5. During this operation mode, the supercapacitor $C_{m}$ charges inductor

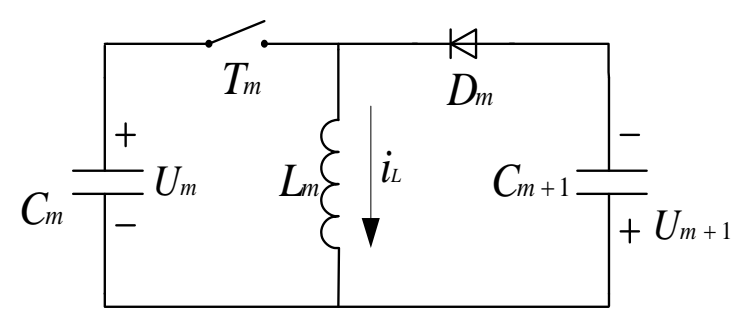

Figure 4. The equivalent circuit of active voltage equalization system

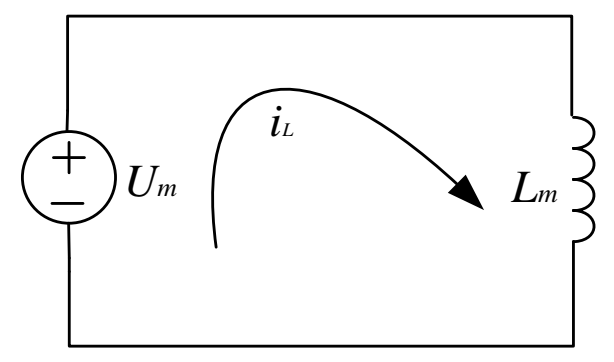

Figure 5. Equivalent circuit at mode 1

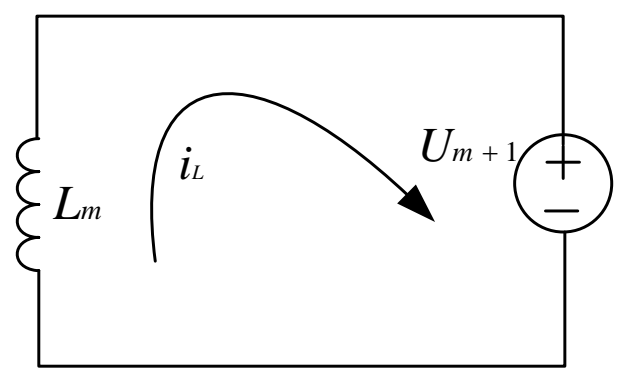

Figure 6. Equivalent circuit at mode 2 
$L_{m}$, and energy is stored in inductor $L_{m}$, the inductor current keeps rising linearly. Thus,

$$
L \frac{i_{L}}{D_{1} \times T}=U_{m}
$$

\subsubsection{Operation Mode $2\left(D_{1} T \leq t \leq\left(D_{1}+D_{2}\right) T\right.$ )}

At $t=D_{1} T$, the switch $T_{m}$ is turned off, the diode $D_{m}$ is turned on. The Figure 4 can be equivalent to Figure 6 . During this operation mode, the inductor $L_{m}$ discharges supercapacitor $C_{m}$, energy is transferred to supercapacitor $C_{m}$, the inductor current keeps falling linearly from peak value to zero. Thus,

$$
L \frac{i_{L}}{D_{2} \times T}=U_{m+1}
$$

3.2.3 Operation Mode $3\left(\left(D_{1}+D_{2}\right) T \leq t \leq T\right)$

In this operation mode, the switch $T_{m}$ and the diode $D_{m}$ are all turned off.

\section{Design and Simulation of Active Voltage Equalization Circuit}

\subsection{Design of the Active Voltage Equalization Circuit Parameters and Control Strategy}

\subsubsection{Operation Range of Duty Ratio}

The following conclusion can be obtained from the (10) and (11).

$$
U_{m} \cdot D_{1}=U_{m+1} \cdot D_{2}
$$

When the voltage equalization system works at steady state, the difference between $U_{m}$ and $U_{m+1}$ is very little. Thus, we can think $U_{m}=U_{m+1}=U$, in addition, because the circuit works in discontinuous conduction mode(DCM).

Then, $D_{1}+D_{2}<1, \quad D_{1}=D_{2}$. thus,

$$
D_{1}=D_{2}=D<50 \%
$$

From the above inequation, we can obtain $D<50 \%$.

\subsubsection{Inductor Selection}

In a switching period, the working curve of inductor( $L$ ) current is shown in the Figure 7 . The average current releases from Supercapacitor to the inductor $L$ is:

$$
I_{\text {avg }}=\frac{D \times I_{L}}{2}
$$

where $I_{L}$ is the peak current of inductor, and it can be expressed as below:

$$
I_{L}=\frac{D T \times U}{L}
$$

Substituting (14) into (15), thus, the average current can be calculated as follows:

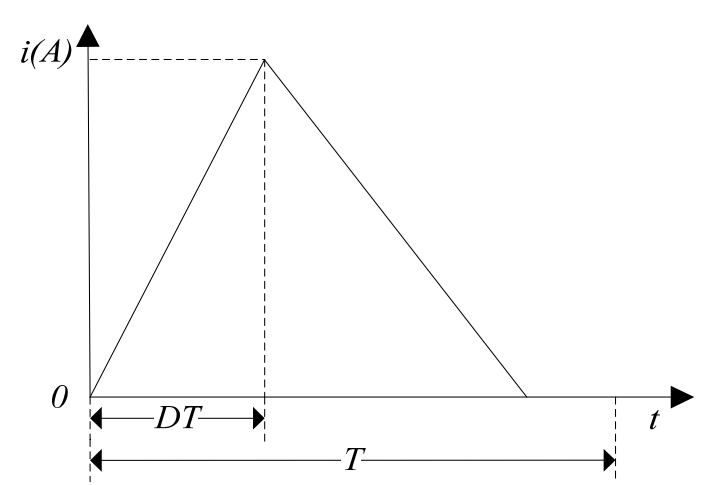

Figure 7. Working curve of inductor current

$$
I_{\text {avg }}=\frac{D^{2} \times T \times U}{2 \times L}
$$

From what has been analyzed, it is considered that the average current of the inductor is also the equalizing current of active voltage equalization circuit. According to the principle of voltage equalization of supercapacitors, if the high-voltage supercapacitors release the average current $I_{\text {avg }}$ more than the equalizing current $I_{e q}$, the voltage equalization can be realized. In order to increase the voltage equalizing speed, select the coefficient $k_{j} \geq 0.2$, then $I_{a v g} \geq 0.2 \times I$, and substituting this in Equation into (16), the inductor $L$ can be obtained as follows:

$$
L \leq \frac{D^{2} \times T \times U}{0.4 \times I}
$$

In the circuit design, once the energy storage inductor $L$ is selected, there are two ways to adjust the switching period:

1) Selecting the fixed switching period $T$, the equalizing current will be restrained by the limited cell voltage $U$ in the process of charging and discharging, which makes it as a function of the voltage $U$;

2) The switching period $T$ changes with the voltage $U$, which makes balancing current become a fixed value.

\subsubsection{Voltage Equalization Control Strategy}

Measuring two supercapacitors voltage $U_{m}$ and $U_{m+1}$, the voltage difference can be calculated by the Equation $\Delta U_{m}=U_{m}-U_{m+1}$, and comparing the difference with the reference voltage $U_{r e f}$, if $\Delta U_{m} \geq U_{\text {ref }}$, then the voltage Equalization circuit begins to work; if $\Delta U_{m} \leq$ $U_{\text {ref }}$, then the voltage equalization circuit stops working. In fact, if make all the supercapacitors reach the voltage equalization, every adjacent two supercapacitors will be parallel connected with a Buck-Boost converter, the voltage equalization controller generates different diving signals by analyzing all the measured supercapacitors 


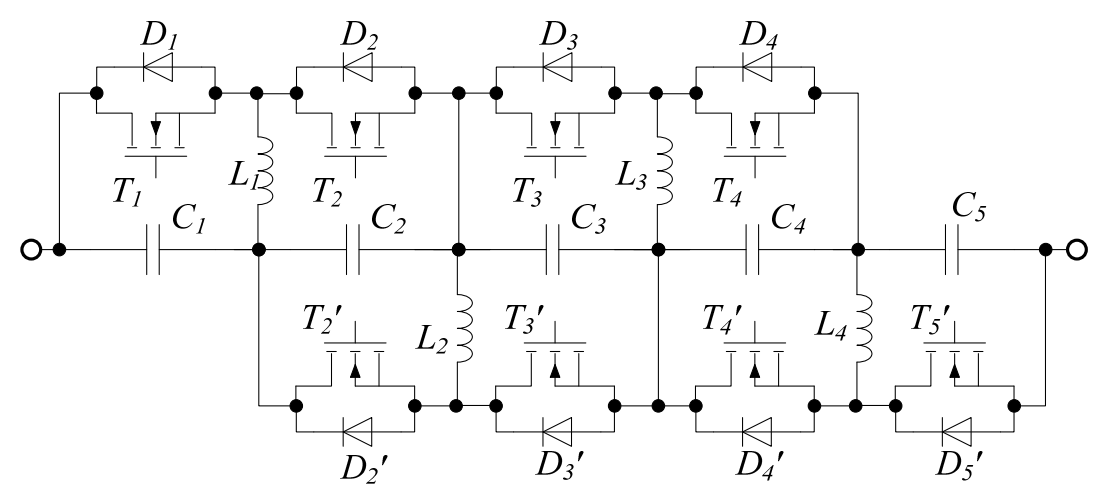

Figure 8. Active circuit of voltages balance for five superca-pacitors series

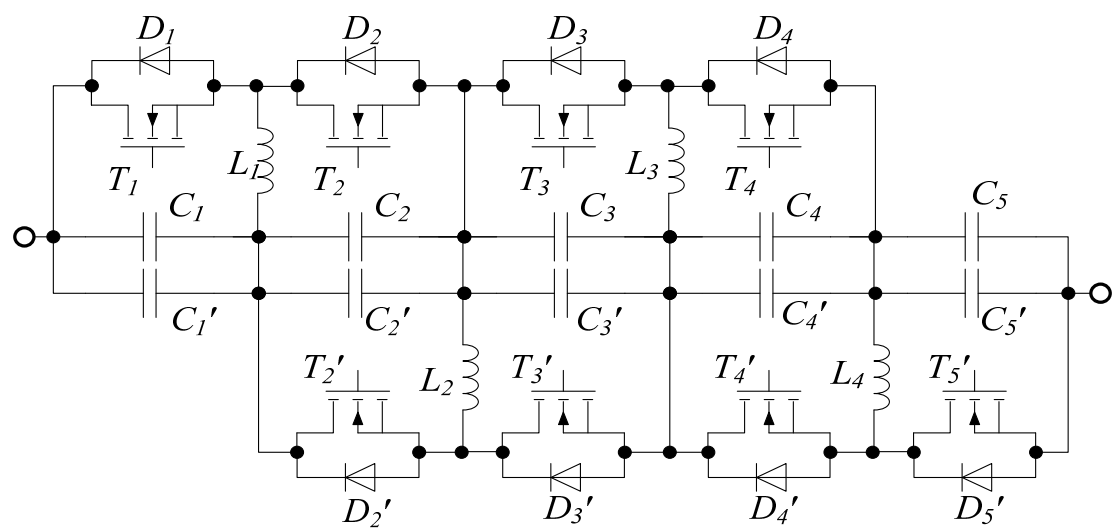

Figure 9. Active circuit of voltages balance for ten supercapacitors

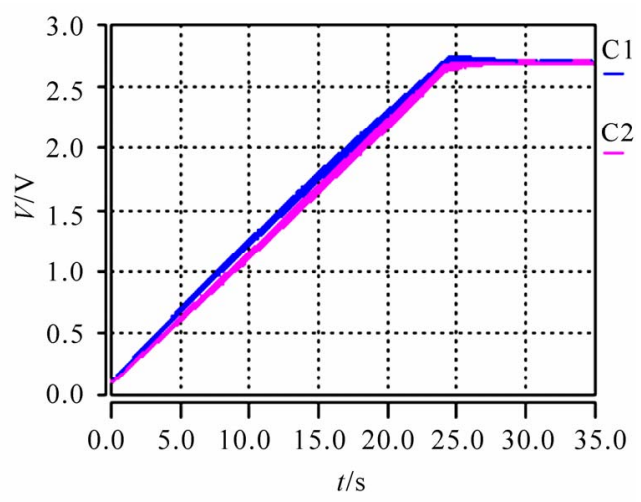

Figure 10. Simulation results of voltages balance for supercapacitors

voltage to drive MOSFET, in this way, equalization among all supercapacitors will be achieved at last.

\subsection{The Extension Circuit of Voltage Equalization}

Extending the above circuit, we can make it suitable for more series supercapacitor cells to meet the requirement of the RTG energy saving system. As is shown in Figure 8 , we can see every two adjacent supercapacitors constitute a group which is controlled to balance voltage by a Buck-Boost converter. For example, the converter, which is composed of $T_{1}, D_{1}, T_{2}$ and $D_{2}$, balances the voltages between $C_{1}$ and $C_{2}$, and another one is composed of $T_{2}, D_{2}, T_{3}{ }^{\prime}$ and $D_{3}{ }^{\prime}$, balances the voltages between $C_{2}$ and $C_{3}$. The principle of equalization among $C_{3}, C_{4}$ and $C_{5}$ is the same as the above [5].

Considering the demand of hundreds of supercapacitor cells in RTG energy saving system, the series structure, as shown in Figure 8, makes the control components increase markedly and the control circuit become complicated. So the series and parallel structure in Figure 9, can be adopted in practical application.

\subsection{Saber Simulation Analysis}

In order to verify the character of the active voltage balance circuit in the energy recycling RTG system, we research the circuit composed of two supercapacitor cells in series module by the simulation study of Saber. Assume that the capacitance of one supercapacitor is 
$800 \mathrm{~F}$ and the other one is $1000 \mathrm{~F}$, the constant charging current is $100 \mathrm{~A}$, the rate voltage of the supercapacitor is $2.7 \mathrm{~V}$, the energy storage inductor is $1.36 \mathrm{uH}$ and the switching frequency is $10 \mathrm{kHz}$.

Figure 10 describes the process of charging two supercapacitors. It can be seen, at the end of the process, that the voltages of two supercapacitors become the same, no over-voltage. The result of Saber simulation indicates that active voltage balance circuit amends the inconsistency of the supercapacitor voltage greatly.

\section{Conclusions}

The active voltage equalization circuit based on the reversible Buck-Boost converter has been discussed in this paper. Theoretical analysis and simulation result show that the active control circuit can better solve the problem of the partial over-voltage over the supercapacitor groups. This method can be applied in the situation of higher charging or discharging current. Therefore, it has a high value to be used in the RTG energy saving system.

\section{REFERENCES}

[1] M. Okamura, "A basic study on power storage systems (translation of Japanese paper by the author with updating comments),’Journal Electrical Engineering in Japan, Vol.115, No.3, pp. 40-51, 1996.

[2] J. Zhao, J. C. Jiang, and L. Y. Niu, “A novel charge equalization technique for electric vehicle battery system," The Fifth International Conference on Power Electronics and Drive Systems, Singapore, 2003.

[3] S. W. Moore and P. J. Schneider, “A review of cell equalization methods for lithiumion and lithium polymer battery systems," SAE Technical Paper Series, pp. 1-5, 2001.

[4] H. D. Li, Z. Y. Feng, and Z. P. Qi, "Study on a fast voltage balancing for supercapacitors," Chinese Journal of Power Sources, Vol. 37, No. 3, pp. 186-189, 2007.

[5] A. Rufer and P. Barrade, “A supercapacitor-based energy-storage system for elevators with soft commutated interface,” IEEE Transactions on Industry Applications, Vol. 38, No. 5, pp. 1151-1159, 2002. 